\title{
Frequency dependence of estimated fatigue limit by lock-in infrared thermography for commercially pure $\mathrm{Ti}$
}

\author{
by T. Matsunaga, T. Nojima*, N. Nagashima
}

National Institute of Materials Science (NIMS), 1-2-1 Sengen Tsukuba, 305-0047, Ibaraki, Japan. MATSUNAGA.Tetsuya@nims.go.jp, NOJIMA.Takehiro@nims.go.jp, NAGASHIMA.Nobuo@nims.go.jp.

\begin{abstract}
Fatigue limit was estimated by the lock-in infrared thermography for commercially pure titanium (CP-Ti JIS Gr. 2) at room temperature. Fatigue tests were performed at low frequencies of $1 \mathrm{~Hz}$ and $4 \mathrm{~Hz}$ at stress ratio $(R)$ of -1 , and $7 \mathrm{~Hz}$ at $R=0.05$. CP-Ti showed valuable dissipated energy at the low frequencies and frequency dependency of fatigue limit was negligible. In addition, a positive correlation is observed between dissipated energy and strain energy. It meant that the increase of dissipated energy results from the increase of plastic deformation.
\end{abstract}

\section{Introduction}

Measurement of fatigue limit for metals or mechanical components is extremely time consuming and expensive statistical process in industries because it often takes many thousands of cycles until failure and large number of specimens are needed. Especially, aerospace industry needs a long developing period because of increasing system complexity [1]. In general, an estimation of fatigue limit of a new material takes more than a year by standard fatigue tests. Because the lock-in infrared thermography is expected to estimate a fatigue limit at $10^{7}$ cycles by single test [2-5], time and cost of safety evaluation can be reduced drastically. To estimate a fatigue limit by the method, dissipate energy $(q)$ was evaluated. The energy might be generated by irreversible temperature change $\left(\Delta T_{D}\right)$ by energy dissipation:

$$
q=C \rho \Delta T_{D}
$$

where $C$ is a specific heat $(0.523[\mathrm{~J} / \mathrm{g} \cdot \mathrm{K}])$ and $\rho$ is a density of the sample $\left(4.51 \cdot 10^{6}\left[\mathrm{~g} / \mathrm{m}^{3}\right]\right)$. Using the method, one or two days are enough to estimate the fatigue limit of a sample. Furthermore, since this method can show a failure location which is pointed as high temperature area on infrared images, its application for product-level inspection is also expected [5-6].

The rapid estimation of fatigue limit has been applied to the specific materials such as carbon steels and stainless steels. It is well known that their values are well coincided with those determined by conventional fatigue tests [7]. Furthermore, aluminum alloys have been investigated with this method because they are common materials in automobile and aerospace industries [5, 9]. However, the method has been less applied for CP-Ti and Ti alloys in spite of common material in aircrafts due to light weight, excellent corrosion resistance and mechanical property. Therefore, the rapid evaluation of fatigue limit was applied for CP-Ti in the present study. This study also attempted to reveal frequency dependence of a fatigue limit to widen applicability of the method.

\section{Experimental procedure}

\subsection{Specimen}

CP-Ti JIS Gr. 2 was used in this study, which chemical composition and mechanical properties are reported in a mill sheet as shown in Tables 1 and 2, respectively. Grain size was evaluated as about $20 \mu \mathrm{m}$. Sample was a plate-type specimen with a thickness of $3 \mathrm{~mm}$ and a width of $3 \mathrm{~mm}$ as shown in Fig. 1. They were manufactured from a round bar with $\phi=12 \mathrm{~mm}$. Surfaces were polished by \#600 emery paper and one surface was matte-coated to eliminate metallic luster.

Table 1. Chemical composition of CP-Ti JIS Gr. 2 (mass \%).

\begin{tabular}{|c|c|c|c|c|c|}
\hline $\mathrm{H}$ & $\mathrm{O}$ & $\mathrm{N}$ & $\mathrm{Fe}$ & $\mathrm{C}$ & $\mathrm{Ti}$ \\
\hline 0.005 & 0.11 & $<0.01$ & 0.10 & 0.01 & Bal. \\
\hline
\end{tabular}

Table 2. Mechanical property of the investigated material.

\begin{tabular}{|c|c|c|c|c|}
\hline $0.2 \%$ proof stress & Tensile stress & Elongation & Reduction of area & Hardness \\
\hline $325[\mathrm{MPa}]$ & $426[\mathrm{MPa}]$ & $31.1[\%]$ & $68.9[\%]$ & $176[\mathrm{HBM}]$ \\
\hline
\end{tabular}




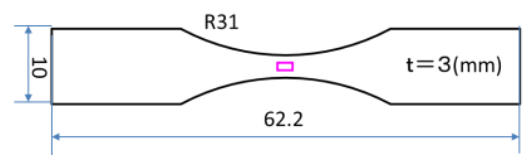

Fig. 1: Geometry of sample for the lock-in infrared thermography.

\subsection{Measurement}

Fatigue tests were conducted at room temperature. Frequencies were $1 \mathrm{~Hz}$ and $4 \mathrm{~Hz}$ at $R=-1$, and $7 \mathrm{~Hz}$ at $R=0.05$. A sine wave loading cycle was applied. The range of stress amplitude were 100-340 MPa for the tests at $R=-1,20-190$ $\mathrm{MPa}$ at $R=0.05$. The range of stress amplitude were $100-340 \mathrm{MPa}$ for the tests at $R=-1,20-190 \mathrm{MPa}$ at $R=0.05$.

A surface of the specimen was observed by an infrared camera installed in front of a fatigue machine. The infrared camera was one of the FLIR-SC7000 series with an accuracy of $0.02 \mathrm{~K}$. In the lock-in analyses, a frame rate of the camera was $99 \mathrm{~Hz}$ and a shooting time was $30.3 \mathrm{~s}$ to obtain 3000 pictures. The $\Delta T_{D}$ measurements were performed three times at a stress amplitude and observed using a software (CERIP Inc. Altair LI). Then, the dissipate energy was evaluated by eq. (1). In the calculation, $\Delta T_{D}$ was average value around the center of specimen. The dissipated energy increased with increasing stress amplitude, and there were two regions with different increasing rates. Fatigue limit of the sample was estimated at a transition point of the $q_{D}$ value at a stress amplitude.

In addition, a strain gauge was attached to measure the stress-strain hysteresis loops. Both voltage signals from strain gauge-amplifier system and from load cell were measured and recorded by the data logger with its $1 \mathrm{kHz}$ logging rate for the tests at $1 \mathrm{~Hz}$, and $2 \mathrm{k} \mathrm{Hz}$ logging rate for the tests at $4 \mathrm{~Hz}$ and $7 \mathrm{~Hz}$. Strain energy was determined by the calculating their hysteresis loop area to compare with the dissipated energy.

Standard fatigue tests were performed at $R=0.05$ and 0 at room temperature to compare the fatigue limit observed by the infrared camera. Frequency range in the tests was $1 \mathrm{~Hz}-30 \mathrm{~Hz}$. Specimen was round-bar type with $\Phi=3 \mathrm{~mm}$.

\section{Experimental Results and Discussion}

\subsection{Dissipated energy}

Fig. 2 shows the dissipated energy functioned by stress amplitude for each frequency at $R=-1$. The energy at 1 $\mathrm{Hz}$ is smaller than that at $4 \mathrm{~Hz}$. It revealed that the low frequency such as $1 \mathrm{~Hz}$ is enough to observe the irreversible temperature change in CP-Ti. In addition, the fraction of the energy became obvious, which might result from the thermal diffusion to air, jig, and so on during the cyclic loading because there is enough time to energy dissipation. The estimated fatigue limits were almost same at both frequencies, i.e., about $250 \mathrm{MPa}$, and the frequency dependency is negligible.

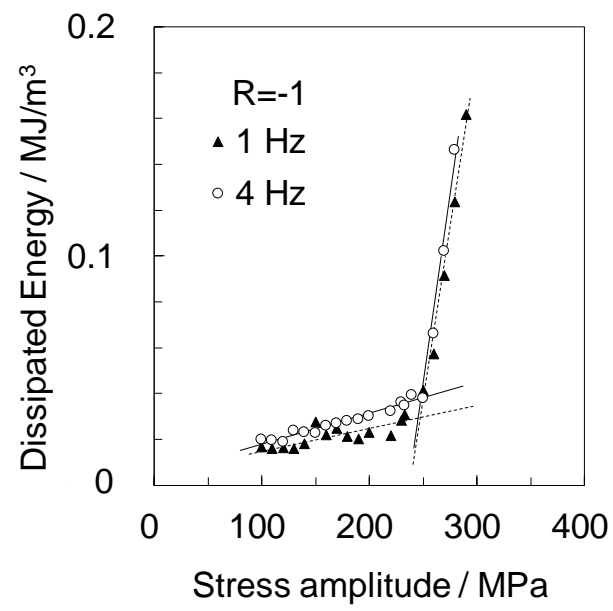

Fig. 2: Relation between dissipated energy and stress amplitude at $R=-1$.

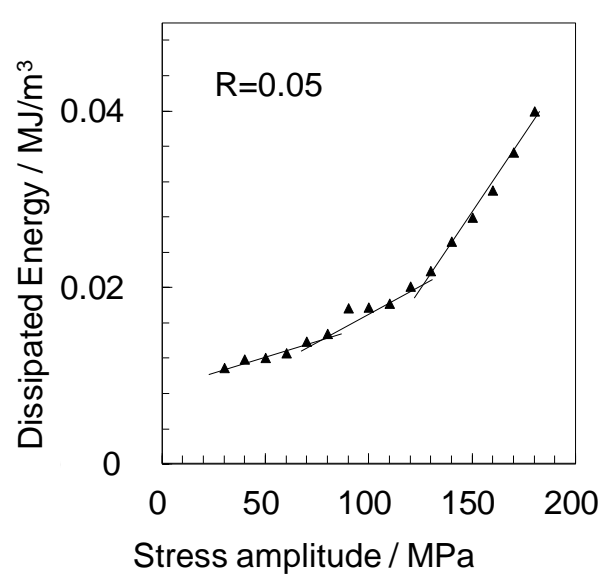

Fig. 3: Relation between dissipated energy and stress amplitude at $R=0.05$. 
Fig. 3 shows a relation between dissipated energy and stress amplitude at $R=0.05$ with $7 \mathrm{~Hz}$. The dissipated energy was small in comparison with that at $R=-1$, but it was well measured even at low stress amplitude due to increased testing frequency which prevent the effect of heat conduction [10]. In addition, the dissipated energy gradually increased by increase of stress amplitude, and two transition points were included. Although the difference of two points will be discussed in sec. 3.2, the estimated fatigue limit was estimated as ca.130 MPa.

Standard fatigue tests were performed at $R=0.05$ and 0 to confirm the fatigue limit estimated using the infrared thermography. A frequency range of the fatigue tests was 1-30 Hz. S-N curve at the conditions was plotted in Fig. 4. The frequency dependency might be negligible because the plotted data did not show remarkable dispersion. In addition, the fatigue limit by the standard fatigue tests might be about $140 \mathrm{MPa}$ because the sample tested at the stress was not ruptured at $10^{7}$ cycles. The stress was almost same as the fatigue limit estimated using the infrared thermography. Therefore, it can be mentioned that the lock-in infrared thermography estimate the fatigue limit of CP-Ti using only one specimen and it is available for the quick safety evaluation.

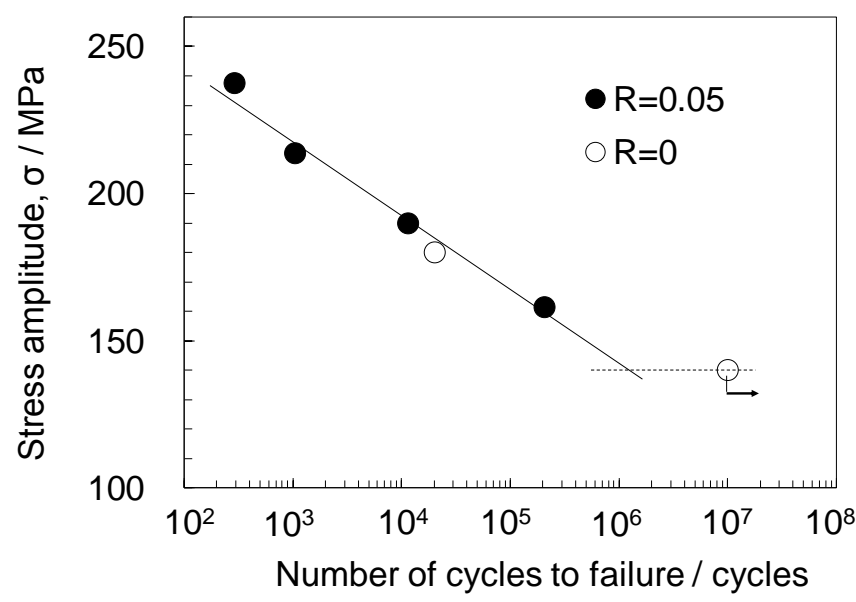

Fig. 4: $S-N$ curve of CP-Ti at $R=0.05$ and 0 at room temperature (297 K).

\subsection{Plastic energy and its relation with dissipated energy}

As mentioned above, the thermography was performed three times with measurement of strain-stress hysteresis loops at given stress amplitudes. Each measurement term has a beginning loop and an ending loop. Fig. 5 shows the examples of hysteresis loops at $R=0.05$ with an enlarged area of their central part. Beginning loops and ending loops at the third measurement are plotted in this figure. At low stress amplitude, the loops are almost overlapped, whereas they get separated into two loops at high stress amplitudes. According to the condition, plastic strain energy was estimated not by area of the hysteresis loop but by envelope area made by beginning loop and ending loop in this study (Fig. 6).

Fig. 7 shows the plastic strain energy functioned by stress amplitude at $R=0.05$. This condition has two transition points. When the loops overlapped as shown in Fig. 5, plastic deformation and its energy were small. Next, the loops did not widen obviously but separated, i.e., plastic strain began to generate, at more than $100 \mathrm{MPa}$ which is corresponding to the transition point at low stress amplitude. Finally, the transition point at high stress amplitude became about $135 \mathrm{MPa}$, which is similar value with the fatigue limit evaluated by this method and conventional fatigue test (140 MPa). At the high stress amplitudes, the loops became widen and remarkable plastic strain stored in the sample. The difference might occur by cyclic softening during fatigue test, which is shown in NIMS fatigue data sheet of CP-Ti [11]. Moreover, the data sheet indicated that cyclic hardening occurs at more than a proof stress. Therefore, the phenomenon related to dislocation motion might affect the generation of dissipated energy; straightly aligned dislocation at low stress [12] and tangled dislocation at high stress. According to the discussion, the high stress amplitude leads to failure and shows the fatigue limit in a finite time because of the clearer strain accumulation comparing with the low stress amplitude. Fig. 8 shows relation between plastic strain energy and stress amplitude at $R=-1$ with $1 \mathrm{~Hz}$ and $4 \mathrm{~Hz}$. The figure showed similar behavior to that at $R=0.05$, whereas the energies were relatively small before the first transition point. On the other hand, the second transition points observed at $1 \mathrm{~Hz}$ and $4 \mathrm{~Hz}$ were used as fatigue limit, i.e., about $250 \mathrm{MPa}$.

Fig. 9 shows a relationship between the dissipated energy and the plastic strain energy for all conditions $(R=-1$ at $1 \mathrm{~Hz}$ and $4 \mathrm{~Hz}$, and $R=0.05$ at $4 \mathrm{~Hz}$ ). As shown in the figure, a positive correlation seems to be observed between them. However, the data at $R=-1$ with $1 \mathrm{~Hz}$ fluctuated more than that at the other conditions. The correlation analysis between dissipated energy and plastic strain energy was calculated, and the results are shown in Table 3. Although the correlation coefficient of the data at $R=-1$ with $1 \mathrm{~Hz}$ at the low range of stress amplitude is relatively weak, there are strong correlation between the both energy at other range and conditions. In addition to the discussion in the previous paragraph, this result also means that the increase of plastic strain energy is one of the factors for the increase of dissipated energy. Therefore, 
the dissipated energy at $1 \mathrm{~Hz}$ should be larger than that at $4 \mathrm{~Hz}$ because the plastic strain energy at $1 \mathrm{~Hz}$ was larger than that at $4 \mathrm{~Hz}$ (see Fig. 8). This result means that the observed dissipated energy at $1 \mathrm{~Hz}$ was reduced due to thermal diffusion (sec. 3.1). Here an energy loss at $1 \mathrm{~Hz}$ was estimated by the following processes. First, a ratio of plastic strain energies at both conditions $\left(q_{1} \mathrm{~Hz} / q_{4} \mathrm{~Hz}\right)$ was calculated at each stress amplitude. Next, the dissipated energy at $1 \mathrm{~Hz}$ was corrected by multiplying the ratio and the dissipated energy at $4 \mathrm{~Hz}$ assuming that there is no energy loss in the energy at $4 \mathrm{~Hz}$. Then, an estimation for the loss of dissipated energy at $1 \mathrm{~Hz}$ was conducted by $\left(q^{\prime}{ }_{1} \mathrm{~Hz}-q_{1} \mathrm{~Hz}\right) / q_{1}^{\prime} \mathrm{Hz}$, where $q^{\prime}{ }_{1} \mathrm{~Hz}$ is the corrected energy at $1 \mathrm{~Hz}$. Fig. 10 shows the relation between the energy loss and stress amplitude. In this figure, the loss is about $10-30 \%$ at low stress amplitude and about $50 \%$ at high stress amplitude. Although the frequency of $1 \mathrm{~Hz}$ is enough to estimate fatigue limit, the loss in measuring dissipated energy is remarkable. The corrected dissipated energy $q^{\prime}{ }_{1} \mathrm{~Hz}$ is plotted against the plastic strain energy with measured the energy $q_{1} \mathrm{~Hz}$ in Fig. 11, where the correlation between the energies at $1 \mathrm{~Hz}$ is improved by the correction of the energy. Furthermore, plastic strain energy might be estimated by this correlation obtained in this study without measuring the hysteresis loop of specimens.

\section{Conclusions}

In the present study, the lock-in infrared thermography was applied for CP-Ti JIS Gr. 2 at room temperature. The fatigue limits were estimated by the new method, and the present authors reveal the frequency dependence of fatigue limit and the relation between dissipated energy and plastic strain energy. The following conclusions are revealed.

(1) The fatigue limits estimated by the thermography were about $250 \mathrm{MPa}$ at $R=-1$, and about $130 \mathrm{MPa}$ at $R=0.05$ at room temperature. The latter value was almost corresponded to that evaluated by the standard fatigue tests.

(2) The frequency dependency of the fatigue tests was negligible at the frequencies of $1 \mathrm{~Hz}-4 \mathrm{~Hz}$.

(3) The dissipated energy at $R=0.05$ was relatively small compared to that at $R=-1$. However, the energy was able to measure accurately due to high frequency of $7 \mathrm{~Hz}$.

(4) A positive correlation was observed between the dissipated energy and plastic strain energy, which means that the increase of plastic strain energy is one of the factors for the increase of dissipated energy.

The authors thank Mr. S. Sugimoto from JFE Techno-Research Corporation for his data analysis and valuable comments. The authors are grateful for fundamental supports from the Innovative Science and Technology Initiative for Security, ATLA, Japan.
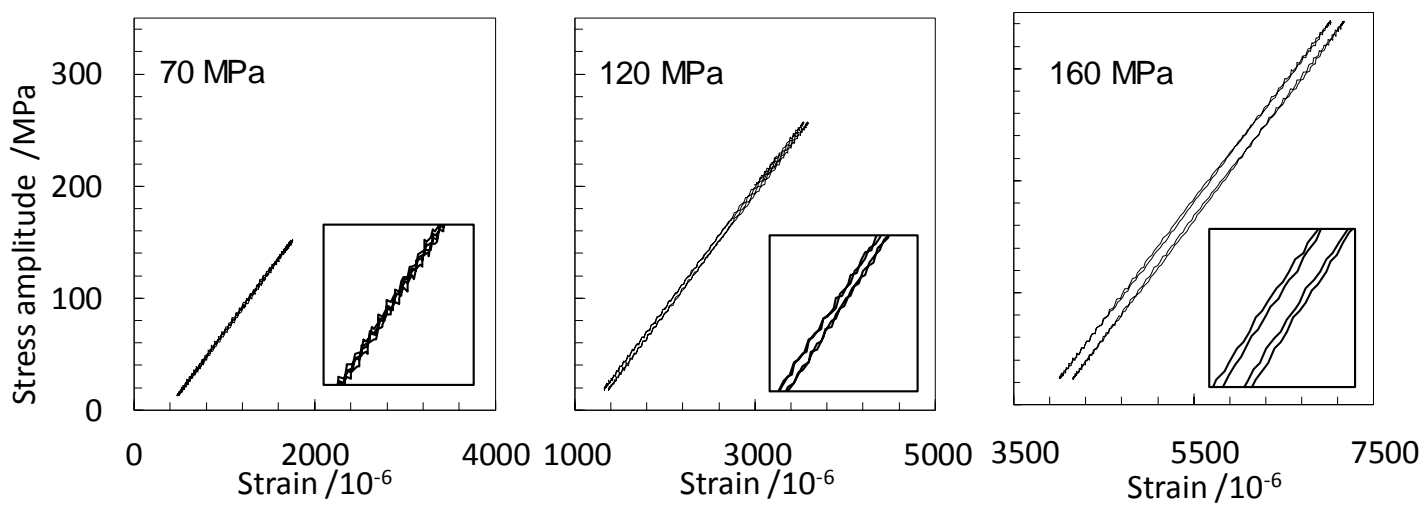

Fig. 5: Series of stress-strain hysteresis loops $(\mathrm{f}=7 \mathrm{~Hz}$ at $R=0.05)$ with enlarged area of their central part.

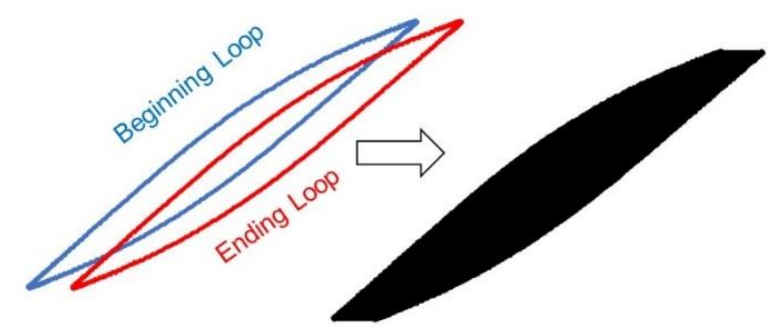

Fig. 6: Schematic drawing of calculated area of strain-stress hysteresis loop for plastic strain energy. 
10.21611/qirt.2019.022

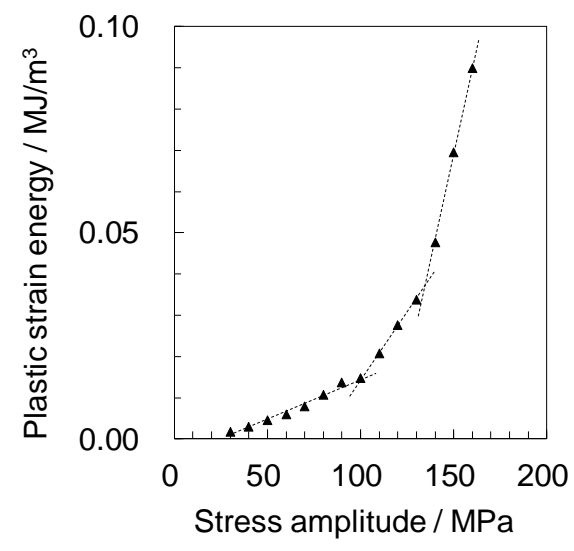

Fig. 7: Relation between plastic energy and stress amplitude at $R=0.05$ at room temperature.

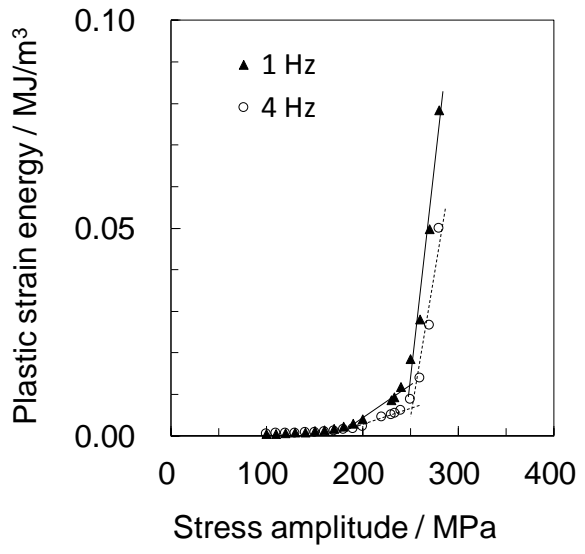

Fig. 8: Relation between plastic energy and stress amplitude at $R=-1$ at room temperature.

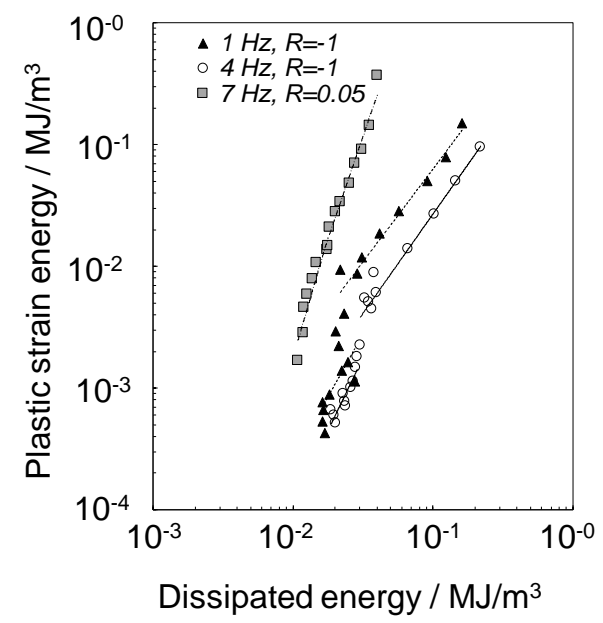

Fig. 9: Relation between plastic energy and the dissipated energy for CP-Ti at room temperature.

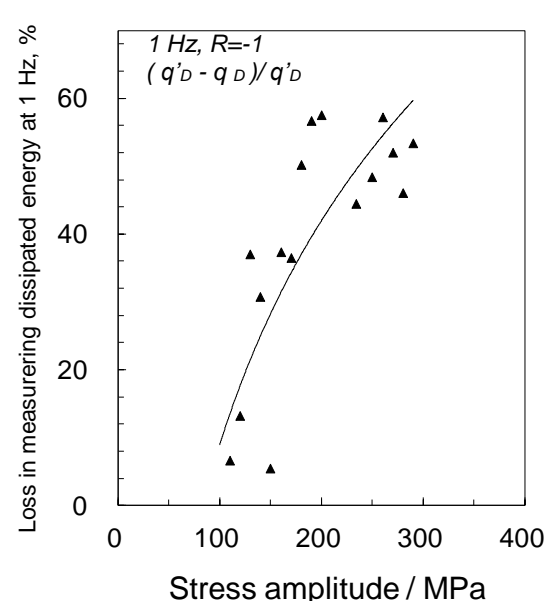

Fig. 10: Relation between loss in measuring dissipate energy and stress amplitude at $1 \mathrm{~Hz}, \mathrm{R}=-1$.

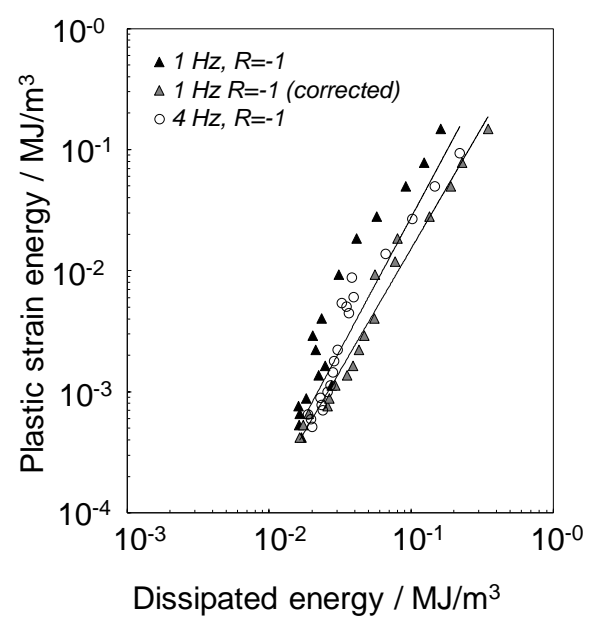

Fig. 11: Relation between plastic energy and the dissipated energy at $R=-1$ for $C P$-Ti at room temperature. Where, the dissipated energy at $1 \mathrm{~Hz}$ with correction and without correction are plotted. 
Table 3. The outcomes of correlation analysis between dissipated energy and plastic energy.

\begin{tabular}{|c|c|c|c|c|c|c|}
\hline & \multicolumn{4}{|c|}{$R=-1$} & \multirow{2}{*}{\multicolumn{2}{|c|}{$\begin{array}{c}\mathrm{R}=0.05 \\
7 \mathrm{~Hz}\end{array}$}} \\
\hline & \multicolumn{2}{|c|}{$1 \mathrm{~Hz}$} & \multicolumn{2}{|c|}{$4 \mathrm{~Hz}$} & & \\
\hline & $\begin{array}{c}\text { calculated } \\
\text { range } \\
\text { [Mpa] }\end{array}$ & $\begin{array}{c}\text { correlated } \\
\text { coefficient } \\
{[\%]}\end{array}$ & $\begin{array}{c}\text { calculated } \\
\text { range } \\
\text { [Mpa] }\end{array}$ & $\begin{array}{c}\text { correlated } \\
\text { coefficient } \\
{[\%]}\end{array}$ & $\begin{array}{c}\text { calculated } \\
\text { range } \\
\text { [Mpa] }\end{array}$ & $\begin{array}{c}\text { correlated } \\
\text { coefficient } \\
{[\%]}\end{array}$ \\
\hline Low $\sigma$ & $100-240$ & 0.48 & $100-240$ & 0.99 & $30-100$ & 0.98 \\
\hline all $\sigma$ & $100-290$ & 0.97 & $100-290$ & 0.91 & $30-180$ & 0.85 \\
\hline all $\sigma$ & $100-290$ & 0.84 & $100-290$ & 0.91 & $30-180$ & 0.97 \\
\hline
\end{tabular}

\section{REFERENCES}

[1] G. Young, Boeing 2013 Industry Symposium.

[2] M.P. Luong. Fatigue limit evaluation of metals using an infrared thermographic technique. Mechanics of materials. 1998 May; 28:155-163.

[3] G. La Rosa, A. Risitano. Thermographic methodology for rapid determination of the ffatigue limit of materials and mechanical components. International Journal of Fatigue. 2000 June ; 22: 65-73.

[4] D. Shiozaki, T. Inagawa, T. Washio, T. Sakagami. Fatigue limit estimation of stainless steels with new dissipated energy data analysis. Proceedia Structural Integrity. 2016 June; 2: 2091-2096.

[5] A. Akai, D. Shiozawa, S. Funazo, K. Chizaka, T. Sakagami. Fatigue strength evaluation for aluminium alloy A6061-T6 based on dissipated energy measurement. Journal of the Society of Materials Science, Japan. 2018 December; 67:1036-1041.

[6] A. Akai, K. Inaba, D. Shiozawa, T. Sakagami. Estimation of fatigue crack initiation location based on dissipated energy measurement. Journal of the Society of Materials Science, Japan. 2015 August; 64: 668-674.

[7] T. Yaoita. Application of thermography to measurement of stress and detection of fatigue limit using dissipated energy. Journal of the Japanese Society for Non-Destructive Inspection. 2002 June; 51(6):333-337.

[8] K. Hayabusa, K. Inaba, H. Ikeda, K. Kishimoto. Estimation of fatigue limits from temperature data measured by IR thermography. Experimental mechanics. 2017 Feburary; 57 (2):185-194.

[9] A. E. Morabito, A. Chrysochoos, V. Dattoma et al. Analysis of heat sources accompanying the fatigue of 2024 T3 aluminium alloys. International Jounal of Fatigue. 2007 May; 29 (5): 977-984.

[10] K. Ryogo, Y. Kurokawa, Y. Irie, H. Inoue. Effect of heat conduction on rapid evaluation of fatigue limit based on temperature variation measurement. 15 th Asia Pacific Conference for Non-Destructive Testing (APCNDT2017).

[11] Data sheet on fatigue properties of pure titanium. NIMS fatigue data sheet No 95. 2004 March.

[12] T. Matsunaga, T. Kameyama, K. Takahashi, E. Sato, Intragranular deformation mechanisms during ambienttemperature creep in hexagonal close-packed metals. Materials Transactions: 2009 November; 60(12): 28652872. 